\title{
Association of Postpartum Pain Sensitivity and Postpartum Depression: A Prospective Observational Study
}

\author{
Yu Chen $\cdot$ Xin Ye $\cdot$ Han $\mathrm{Wu} \cdot$ Xinlei Huang $\cdot$ Congrong Ke \\ Yanqing Chen $\cdot$ Huanghui Wu (D) Xiaodan Wu
}

Received: June 28, 2021 / Accepted: September 8, 2021 / Published online: September 27, 2021

(C) The Author(s) 2021

\begin{abstract}
Introduction: Postpartum depression (PPD) is a severe psychiatric disorder that negatively affects postnatal mothers worldwide. The aim of the study is to investigate the association between postpartum pain sensitivity and PPD as well as to identify the other potential risk factors for PPD and their interconnections.
\end{abstract}

Yu Chen and Xin Ye contributed equally to this work.

Supplementary Information The online version contains supplementary material available at https:// doi.org/10.1007/s40122-021-00325-1.

Y. Chen $\cdot$ H. Wu $\cdot$ Y. Chen $\cdot$ X. Wu $(\bowtie)$ Department of Anesthesiology, Jinshan Branch of Fujian Provincial Hospital, No. 516, South of Jinrong Rd, Fuzhou 350028, China e-mail: wxiaodan@sina.com

Y. Chen $\cdot$ X. Ye $\cdot$ X. Huang $\cdot$ Y. Chen $\cdot$ X. Wu Department of Anesthesiology, Fujian Provincial Clinical Medical College, Fujian Medical University, Fuzhou 350001, China

X. Ye $\cdot$ X. Huang

Department of Anesthesiology, Fujian Provincial

Hospital, Fuzhou 350001, China

C. Ke

Department of Obstetrics and Gynecology, Jinshan Branch of Fujian Provincial Hospital, Fuzhou 350028, China
Methods: The current study was a prospective observational clinical study that enrolled 210 singleton pregnant women in a tertiary referral hospital from January to December 2020. Postpartum pain sensitivity was assessed by the Mandarin Chinese version of the Pain Sensitivity Questionnaire (PSQ), and PSQ-C-total and PSQ-C-minor scores were collected. PPD was screened by self-reporting Edinburgh Postnatal Depression Scale (EPDS), and EPDS total score was recorded. The association between postpartum pain sensitivity and PPD, as well as the risk factors and their interconnections, was analyzed by using a combination of stratified, bivariate, correlation, receiver-operating

\footnotetext{
H. Wu $(\bowtie)$

Department of Anesthesiology, Xiang'an Hospital of Xiamen University, School of Medicine, Xiamen University, No. 2000, East of Xiang'an Rd, Xiamen 361102, China e-mail:mzkwhh@126.com

H. Wu

Department of Anesthesiology and Perioperative Medicine, 900 Hospital of the Joint Logistics Team of the PLA, Fuzhou 350025, China
} 
characteristic (ROC) curve, multivariate logistic regression, and path analysis.

Results: Overall, 87 of 210 mothers (41.4\%) screened positive for PPD. A positive correlation between PSQ-C-total $(r=0.67)$ or PSQ-C-minor $(r=0.62)$ and EPDS scores was identified. PSQC-total [area under curve (AUC) $0.89,95 \%$ CI 0.84-0.93; $P<0.0001]$ showed significantly higher diagnostic accuracy compared with PSQC-minor (AUC 0.85, 95\% CI 0.80-0.90; $P<0.0001)$ in predicting EPDS scores $\geq 10$ points. Multivariate logistic regression indicated that a high PSQ-C-total score [adjusted odds ratio (OR) 1.12, 95\% CI 1.08-1.51; $P<0.001]$ and gestational diabetes (adjusted OR 2.68, 95\% CI 0.96-7.47; $P=0.045$ ) were independent risk factors, while breastfeeding (adjusted OR: 0.34, 95\% CI 0.15-0.78; $P=0.007$ ) and normal birth weight (adjusted OR 0.17 , 95\% CI 0.05-0.61; $P=0.0047$ ) were independently associated with decreasing adjusted ORs for EPDS scores $\geq 10$ points. The path analysis model indicated that PSQ-C-total scores and gestational diabetes showed bidirectional effects, while birth weight and breastfeeding only had a direct impact on EPDS scores $\geq 10$ points.

Conclusion: Increased postpartum pain sensitivity was closely associated with EPDS scores $\geq 10$ points, and PSQ-C scores could be a reliable predictor. Moreover, gestational diabetes, low occurrence of breastfeeding, and low birth weight were the risk factors for EPDS scores $\geq 10$ points.

Trial Registration: ChiCTR.org.cn identifier, ChiCTR-2000033091.

Keywords: Pain sensitivity; Postpartum depression; Pain sensitivity questionnaire; Edinburgh Postnatal Depression Scale

\section{Key Summary Points}

Postpartum hyperalgesia is common in mothers whether they undergo spontaneous delivery, assisted vaginal delivery, or cesarean section. However, the association between postpartum pain sensitivity and postpartum depression in mothers has still not been revealed.

Eighty-seven of 210 mothers (41.4\%) who screened positive for postpartum depression (Edinburgh Postnatal Depression Scale score $\geq 10$ points) exhibited significantly higher pain sensitivity questionnaire-total and pain sensitivity questionnaire-minor scores than those who screened negative.

Gestational diabetes, low occurrence of breastfeeding, and low birth weight were the risk factors for mothers with Edinburgh Postnatal Depression Scale score $\geq 10$ points.

\section{INTRODUCTION}

Postpartum depression (PPD) is a common and serious mental disorder with sadness, anxiety, upset, insomnia, guilt, irritability, and even suicidal tendency during the post-birth period. PPD not only negatively affects $10 \%$ to $40 \%$ of postnatal women worldwide, contributing to one in five mothers' deaths $[1,2]$, but also their caregivers, infants, siblings, and entire families $[3,4]$. Epidemiological data report that the prevalence of PPD ranges from 15 to $20 \%$ in mainland China, and this translates into approximately 5-7 million Chinese women with PPD according to pregnancy and birth records reported by the National Bureau of Statistics of China in 2018. Furthermore, some observational studies from China indicate $>$ $50 \%$ of primiparas may experience a period of unstable moods, such as depressed moods, tearfulness, and mild irritability, and 
accompanying varying degrees of physical symptoms, and may require therapeutic strategies [5-8]. Considering the high disease burden of PPD and its potential severe consequences for mothers and their offspring, as well as their families in China, there is an immense need to identify modifiable risk factors to target preventive and predictive efforts.

In addition to the risk factors, including biological, financial, social, and psychological, that have been reported to contribute to PPD, other medical stresses during the perinatal period are worth considering, especially labor pain and postpartum hyperalgesia. During delivery, uterine contractions, cervical dilatation, and blood interruption lead to stress, tissue damage, and, strikingly, severe pain. Besides, previous studies have suggested that acute postoperative pain that is not well controlled and/or more intense is significantly associated with a higher risk of chronic pain in women undergoing cesarean section [9-11]. Moreover, the intensity of acute pain after delivery has been confirmed to be a critical risk factor for developing postpartum hyperalgesia and persistent pain, whether mothers undergo spontaneous delivery, assisted vaginal delivery, or cesarean section [12]. Notably, evidence from animal and clinical studies indicated that pain sensation could change from acute pain caused by tissue damage to a complex and multifaceted pain syndrome; moreover, the intensity of pain sensitization or hyperalgesia increases over time after surgical or traumatic procedures [13, 14]. Therefore, variation in pain sensitivity can be considered a precursory indicator of developing persistent pain $[15,16]$. Previous studies have reported a bidirectional association between pathological pain and psychological outcomes, such as anxiety and depression, in non-obstetric patients where pain sensitization generally accelerates depressive symptoms, while depression leads to hyperalgesia to pain stimuli $[17,18]$. However, whether postpartum pain sensitivity is associated with postpartum depression in mothers is still unclear. Thus, the aim of the study is to investigate the association between postpartum pain sensitivity and PPD screened by Edinburgh Postnatal Depression Scale (EPDS) scores $\geq 10$ points as well as to identify the other potential risk factors in the perinatal period and their interconnections by using a path model.

\section{METHODS}

\section{Participants}

All the procedures performed in the study involving human participants were in accordance with the ethical standards of the institutional and/or national research committee and with the 1964 Helsinki Declaration and its later amendments or comparable ethical standards. The study protocol was approved by the Institutional Ethics Committee (IEC) of Fujian Provincial Hospital (no. K2020-04-047) and registered on ChiCTR.org.cn (ChiCTR2000033091). All parturients received written and verbal information about the study from researchers or midwives, and they signed written consent forms before participation. All parturients aged $>18$ years with American Society of Anesthesiologists (ASA) physical status II, with singleton pregnancies with gestational age $>36$ weeks, and delivered at the Jinshan Branch of Fujian Provincial Hospital from January 2020 to December 2020 were screened. Exclusion criteria were parturients with (1) history of psychiatric disease including anxiety or depression disorder and those receiving psychotropic medications; (2) chronic or frequent pain conditions (moderate to severe pain for $>5$ days per month); (3) any severe obstetric complications including abruptio placentae or placenta previa; (4) unwilling or unable to fill out the questionnaires.

\section{Postpartum Pain Sensitivity Assessment}

Postpartum pain sensitivity was assessed by a pain sensitivity questionnaire (PSQ) developed as a clinically useful, valid self-rating measurement based on pain intensity ratings in daily life situations [19-21], which helps identify patients at risk of intense acute postoperative pain. PSQ-C is the Mandarin Chinese version of the PSQ, which is practical and reliable for 
assessing pain sensitivity in the Chinese population [22]. Hence, the PSQ-C was applied to evaluate the extent of pain sensitivity in mothers after delivery $[19,23]$. The PSQ-C score includes 17 items rated from 0 (not at all painful) to 10 (most severe pain imaginable) on a numeric rating scale (NRS). The PSQ-C total score contains two subscales (PSQ-C-minor and PSQ-C-moderate) and is calculated as the average rating of all items except three non-painful items. Mean scores of $<4,4-6$, and $>6$ are considered minor, moderate, and severe sensitivities to pain, respectively [20]. According to previous recommendations [20, 23], PSQ-C-total and PSQ-C-minor scores were collected in the current study.

\section{Postpartum Depression Screening}

PPD was screened by a widely used self-reporting EPDS for screening depressive symptoms during pregnancy and the postpartum period with good reliability and validity [24]. EPDS contains a total of ten items, and each item was divided into four grades (0 to 3 ) according to the presence and intensity of PPD. The EPDS total score ranges from 0 to 30 points, and a total score $\geq 10$ is considered positive screening for PPD according to the recommendations $[25,26]$.

\section{Data Collection and Outcomes}

Demographic data including maternal age, body mass index (BMI), parity (primiparous or multiparous), gestational age, smoking history (yes or no), detailed medical history including comorbidities (gestational hypertension or preeclampsia, gestational diabetes, anemia), obstetric conditions including preterm (yes or no), mode of delivery (spontaneous vaginal delivery, forceps- or vacuum-assisted vaginal delivery, or cesarean section), dilation of labor (for spontaneous and induction labor), use of epidural labor analgesia (yes or no), perineal injuries at delivery (grades 1-4), length of hospital stay, neonatal outcomes, and breastfeeding (exclusive or any breastfeeding) were recorded. The postnatal clinic provided postdischarge primary care support for breastfeeding mothers and infants. Breastfeeding was evaluated according to the comprehensive assessment of milk production, lactation performance, breastfeeding comfort, and infant growth and health by obstetricians in the postnatal clinic. Adequate milk supply with a comfortable and good latch during breastfeeding and normal growth and development of the baby are assessed as breastfeeding success. The primary outcomes were PSQ-C at 1 week and EPDS scores at 6 weeks postpartum.

\section{Sample Size Calculation}

According to the recommended subject-to-item ratio of $10: 1[22,27]$, at least 170 participants for the PSQ-C (10 participants for each item for a total of 17 items) were required to be able to detect a strong correlation with $80 \%$ power and $5 \%$ significance. Considering an anticipated item-wise missing rate of $10-20 \%$, approximately 200 cases needed to be enrolled in a testretest analysis and its validity analysis.

\section{Statistical Analysis}

Quantitative data correlating to Gaussian distribution were presented as mean \pm standard deviation $(S D)$, while those with abnormal distribution were presented as median [interquartile range $(I Q R)]$. Normality of data was tested by Kolmogorov-Smirnov one-sample test. Variables according to Gaussian distribution were analyzed using Student's $t$-test, while those with abnormal distribution were analyzed using Mann-Whitney U-test. Categorical variables were presented as number (proportion) and analyzed using the chi-square test or Fisher's exact test as appropriate. Stratified analyses according to the average PSQ-C-minor and PSQC-total scores (mild: $>0$ and $\leq 4$; moderate: $>4$ and $\leq 7$; severe: $>7$ and $\leq 10$ scores) were carried out to verify the severity of postpartum pain sensitivity and the association with occurrence of PPD. Postpartum pain sensitivity was analyzed by using PSQ-C-total and PSQ-Cminor together with demographics, delivery characteristics, and breastfeeding between 
mothers with EPDS score $\geq 10$ or $<10$ points using bivariate analyses. Moreover, the relationship between PSQ-C-total or PSQ-C-minor scores and EPDS was analyzed by Spearman correlation coefficient $(r)$ with scatter plots. The receiver-operating characteristic (ROC) curve was analyzed to determine the ability of PSQ-Ctotal and PSQ-C-minor scores to predict the presence of PPD in all participants. The areas under curves (AUCs) with their 95\% confidence intervals (CIs) were calculated. Comparison of ROC curves was performed using the nonparametric technique according to a previous study to choose better predictors [28]. Multivariate logistic regression analysis using enter selection was performed to identify the factors independently associated with presence of PPD. Furthermore, a path analysis model was performed to verify the association between significant variables in the multivariate logistic regression model and EPDS score $\geq 10$ points. To evaluate the fitness of the hypothetical path model, the following values should be acceptable: (1) ratio of likelihood ratio $\chi^{2}$ values to degree of freedom values (CMIN/DF) less than two times; (2) goodness-of-fit index (GFI), adjusted goodness-of-fit index (AGFI), normed fit index (NFI), relative fit index (RFI), incremental fit index (IFI), Tacker-Lewis fit index (TLI), and comparative fit index (CFI) of all values $\geq 0.900$; (3) root mean square error of approximation (RMSEA) [29]. Statistical analyses were performed by using IBM SPSS Statistics 23.0 (IBM Corp., USA), Python ${ }^{\mathrm{TM}} 3.9 .5$ (available on https://www.python.org), and MedCalc 23.0 (MedCalc Software, Belgium). The path model was established using IBM SPSS AMOS 23.0 (IBM Corp., USA), which was also utilized to draw the path diagram. All $P$-values $<0.05$ (two-tailed) were considered statistically significant.

\section{RESULTS}

A total of 332 parturients were screened, and 35 were excluded according to the exclusion criteria. Moreover, 87 women were excluded because of the loss to follow-up (29 mothers with no response on PSQ-C scores at 1 week, while 58 with no response on EPDS scores at 6 weeks postpartum), leaving a final sample of 210 participants for final analysis (Fig. 1).

Overall, 87 of 210 mothers (41.4\%) screened positive for PPD (EPDS score $\geq 10$ points). Demographics and delivery and postpartum characteristics in women with EPDS scores $\geq 10$ or $<10$ points are presented in Table 1 and Supplementary Material. Mothers with EPDS scores $\geq 10$ points exhibited significantly higher PSQ-C-total and PSQ-C-minor scores than those with EPDS scores $<10$ points (Fig. 2). Correlation analysis also revealed a positive correlation between PSQ-C-minor $(r=0.62,95 \%$ CI 0.52-0.70, $P<0.0001$, Fig. 3A) and PSQ-C-total $(r=0.67,95 \%$ CI $0.58-0.74$, $P<0.0001$, Fig. 3B) and EPDS scores. Moreover, stratified analysis according to the average PSQC-minor (Fig. 4A) and PSQ-C-total (Fig. 4B) scores (mild: $>0$ and $\leq 4$; moderate: $>4$ and $\leq 7$; severe: $>7$ and $\leq 10$ scores) was carried out to verify the severity of postpartum pain sensitivity and their association with EPDS scores $\geq 10$ points. Additionally, bivariate comparisons indicated that mothers with EPDS scores $\geq 10$ points were more likely to have a history of gestational diabetes, a prolonged time of second stage labor, and lower occurrence of breastfeeding (Table 2).

Both PSQ-C-total (AUC 0.89, 95\% CI $0.84-0.93 ; P<0.0001$ ) and PSQ-C-minor (AUC $0.85,95 \%$ CI $0.80-0.90 ; P<0.0001$ ) showed good diagnostic accuracy in predicting EPDS scores $\geq 10$ points according to ROC analysis (Fig. 5). Furthermore, PSQ-C-total presented a significantly higher diagnostic accuracy compared with PSQ-C-minor (AUC PSQ-C-total $^{-}$ AUC $_{\text {PSQ-C-minor: }}$ 0.03, 95\% CI $0.01-0.06$; $P=0.023$.

Further multivariate logistic regression indicated that high PSQ-C-total score [adjusted odds ratio (OR) 1.12, 95\% CI 1.08-1.51; $P<0.001$ ] and gestational diabetes (adjusted OR 2.68, 95\% CI 0.96-7.47; $P=0.045$ ) were independent risk factors for EPDS scores $\geq 10$ points, while breastfeeding (adjusted OR 0.34, 95\% CI $0.15-0.78 ; P=0.007$ ) and normal birth weight (adjusted OR 0.17, 95\% CI 0.05-0.61; $P=0.0047)$ were independently associated with decreasing ORs for EPDS scores $\geq 10$ points 


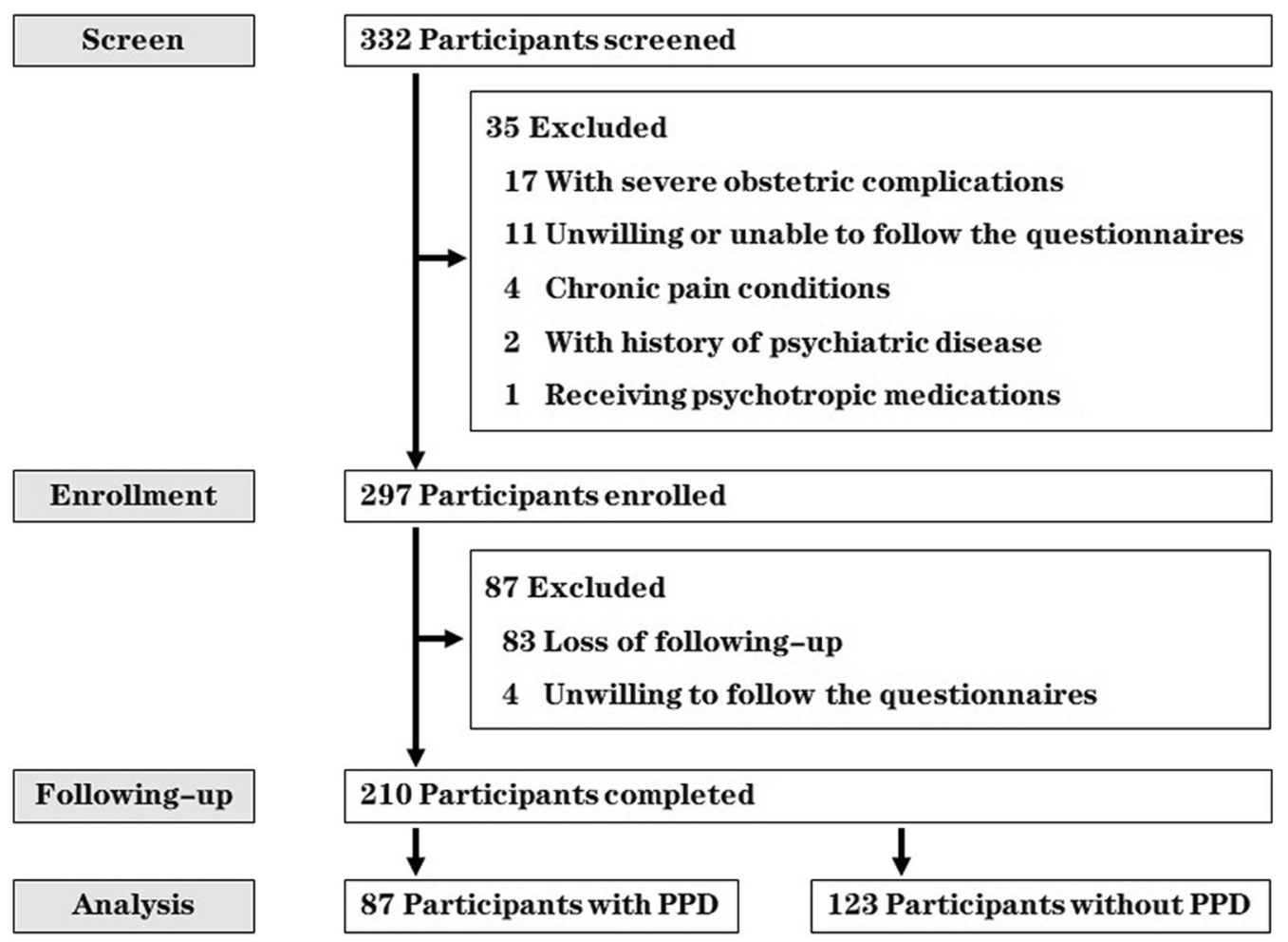

Fig. 1 Overview of participants' screen and enrollment for study

after adjusting for age, prenatal BMI, primiparae, mode of delivery, and labor analgesia (Table 3).

To verify the associations between significant variables in the multivariate logistic regression model and EPDS scores $\geq 10$ points, a path model was constructed (Fig. 6), and the estimates of standardized direct, indirect, and total effects on EPDS scores $\geq 10$ points were further analyzed (Table 4). The results indicated that PSQ-C-total scores not only directly affected PPD (direct effect coefficient: 0.617), but also showed the indirect influence of initiation of breastfeeding (indirect effect coefficient: 0.014). Besides, gestational diabetes also had bidirectional effects on PPD (direct effect coefficient: 0.107; indirect effect coefficient: -0.007 ). However, birth weight (direct effect coefficient: - 0.125) and breastfeeding (direct effect coefficient: - 0.120) only had a direct impact on PPD. The results of fitting indices of this path model indicated that the hypothetical model fit well with sample data $(P=0.924$, $\mathrm{CMIN} / \mathrm{DF}=0.009, \quad \mathrm{NFI}=1.000, \quad \mathrm{RFI}=0.999$,
$\mathrm{IFI}=1.008, \quad$ TLI $=1.126, \quad \mathrm{CFI}=1.000, \quad$ and RMSEA $<0.001)$.

\section{DISCUSSION}

Our study revealed that mothers with EPDS scores $\geq 10$ points exhibited increased PSQ-Ctotal and PSQ-C-minor scores compared with those with EPDS scores $<10$ points, indicating a correlation of postpartum pain sensitivity and PPD, by using a combination of bivariate, correlation, ROC, multivariate logistic regression, and path analysis.

Generally, long-lasting and/or uncontrolled severe acute pain induces hyperalgesia, leading to a chronic and persistent pain condition. Thus, acute pain is no longer a warning signal, but becomes a prediction factor for chronic pain and psychiatric disorders, including anxiety and depression [30]. Evidence from animal studies indicates that alteration of the endogenous modulation system, including a variety of painrelated ion channels, contributes to postpartum 
Table 1 Comparison of demographics and delivery and postpartum characteristics in women with and without a positive postpartum depression screen at the time of a postpartum outpatient clinic

\begin{tabular}{|c|c|c|c|}
\hline \multirow[t]{2}{*}{ Variables } & \multicolumn{2}{|c|}{ Positive PPD screen* } & \multirow[t]{2}{*}{$P$ value } \\
\hline & Yes $(n=87)$ & No $(n=123)$ & \\
\hline \multicolumn{4}{|c|}{ Demographics and antepartum characteristics } \\
\hline Age (years) & $28.5 \pm 3.8$ & $29.0 \pm 3.6$ & 0.637 \\
\hline BMI $\left(\mathrm{kg} / \mathrm{m}^{2}\right)$ & $26.2 \pm 3.9$ & $26.1 \pm 2.9$ & 0.846 \\
\hline Smoking (\%) & $1(1.2)$ & $2(1.6)$ & $>0.999$ \\
\hline Primipara (\%) & $44(50.6)$ & $53(43.1)$ & 0.326 \\
\hline \multicolumn{4}{|l|}{ Maternal disease } \\
\hline Hypertension (\%) & $1(1.2)$ & $3(2.4)$ & 0.644 \\
\hline Gestational diabetes (\%) & $19(21.8)$ & $13(10.6)$ & 0.034 \\
\hline Anemia (\%) & $19(21.8)$ & $20(16.3)$ & 0.368 \\
\hline \multicolumn{4}{|l|}{ Delivery characteristics } \\
\hline Estimated gestational age (weeks) & $39.1 \pm 1.1$ & $39.1 \pm 1.0$ & 0.703 \\
\hline Preterm (\%) & $3(3.4)$ & $1(0.8)$ & 0.309 \\
\hline \multicolumn{4}{|l|}{ Mode of delivery } \\
\hline Spontaneous (\%) & $38(43.7)$ & $65(52.8)$ & 0.276 \\
\hline Assisted vaginal (\%) & $11(12.6)$ & $9(7.3)$ & \\
\hline Cesarean (\%) & $38(43.7)$ & $49(39.8)$ & \\
\hline \multicolumn{4}{|l|}{ Stage of labor (min) } \\
\hline First stage of labor & $497.6 \pm 332.7$ & $448.0 \pm 228.4$ & 0.339 \\
\hline Second stage of labor & $45.3 \pm 40.0$ & $30.2 \pm 27.6$ & 0.016 \\
\hline Total stage of labor & $561.8 \pm 323.1$ & $489.3 \pm 236.8$ & 0.171 \\
\hline Labor analgesia (\%) & $31(35.6)$ & $40(32.5)$ & 0.659 \\
\hline \multicolumn{4}{|l|}{ Perineal injury } \\
\hline I degree & $23 / 49$ & $45 / 74$ & 0.143 \\
\hline II degree & $5 / 49$ & $11 / 74$ & 0.587 \\
\hline III and IV degree & $21 / 49$ & $18 / 74$ & 0.047 \\
\hline Birth weight $(\mathrm{kg})$ & $3.2 \pm 0.4$ & $3.3 \pm 0.4$ & 0.063 \\
\hline Hospital days (d) [IQR] & $4.0[4.0,5.0]$ & $4.0[4.0,5.0]$ & 0.758 \\
\hline \multicolumn{4}{|l|}{ Postpartum characteristics } \\
\hline PSQ-C-total score (point) [IQR] & $81.0[67.0,91.0]$ & $52.0[43.0,62.0]$ & $<0.0001$ \\
\hline PSQ-C-minor score (point) [IQR] & $33.0[25.0,39.0]$ & $19.0[15.0,25.0]$ & $<0.0001$ \\
\hline EPDS score (point) [IQR] & $13.0[11.0,15.0]$ & $6.0[3.0,9.0]$ & $<0.0001$ \\
\hline
\end{tabular}


Table 1 continued

\begin{tabular}{llll}
\hline Variables & \multicolumn{2}{l}{ Positive PPD screen* } & \multicolumn{1}{l}{$\boldsymbol{P}$ value } \\
\cline { 2 - 3 } & Yes $(\boldsymbol{n}=\mathbf{8 7})$ & No $(\boldsymbol{n}=\mathbf{1 2 3})$ & \\
\hline Exclusive breastfeeding (\%) & $69(79.3)$ & $113(91.9)$ & 0.012 \\
\hline
\end{tabular}

A total score $\geq 10$ was considered significant for PPD

$B M I$ body mass index, EPDS Edinburgh Postnatal Depression Scale, IQR interquartile range, PPD postpartum depression, $P S Q-C$ Mandarin Chinese version of pain sensitivity questionnaire

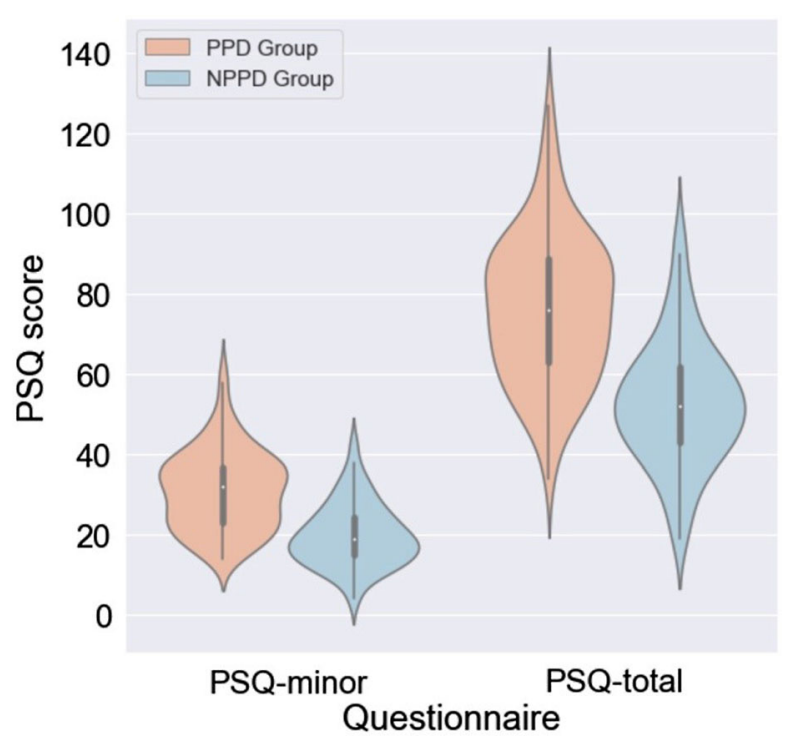

Fig. 2 Violin plots of the PSQ-C scores for mothers with or without PPD

hyperalgesia [31]. Moreover, clinical evidence demonstrates that the severity of acute pain during the perinatal period is a significant predictor of the development of chronic pain, which occurs in $8-22 \%$ of women who undergo either cesarean delivery or vaginal birth [32-34]. Furthermore, mothers with severe acute postpartum pain had a 2.5 -fold increased risk of persistent pain and a 3.0-fold increased risk of developing PPD compared to those with mild postpartum pain, and this negatively affects breastfeeding and infant care [12, 35]. Several clinical studies have also found that uncontrolled acute pain during the peripartum period is closely related to PPD and post-traumatic stress disorder (PTSD) [12, 36, 37]. The intensity of acute pain is a good factor for predicting PPD [27]. However, very little is known about the association between pain sensitivities during postpartum and PPD. Therefore, we introduced the PSQ-C with PSQ-C-total and PSQ-C-minor scores to evaluate postpartum pain sensitivity $[20,23]$; these have been widely used to investigate postoperative pain sensitivity [38-40]. Our results revealed that both PSQ-C-total and PSQ-C-minor scores correlated well with EPDS scores $\geq 10$ points. Furthermore, the ROC analysis indicated that higher diagnostic accuracy was shown in PSQ-C-total compared with PSQ-C-minor scores for predicting EPDS scores $\geq 10$ points. Thus, the PSQ-C-total score was selected as an indicator of postpartum pain sensitivity and included in the following multivariate analysis. The multivariate logistic regression analysis further showed that the PSQC-total score is an independent predictor of EPDS scores $\geq 10$ points, and this association was still significant after adjusting for maternal age, antenatal BMI, parity, delivery mode, and labor analgesia. According to the regression analysis, every 1-point increase of the PSQ-Ctotal score resulted in a $12 \%$ incidence increase of PPD screened according to the EPDS scale. We further identified other significant independent risk factors for EPDS scores $\geq 10$ points including mothers with gestational diabetes and breastfeeding.

To better understand the impact and potential interrelations between these risk factors and EPDS scores $\geq 10$ points, a path analysis model was conducted. This approach allows breaking 


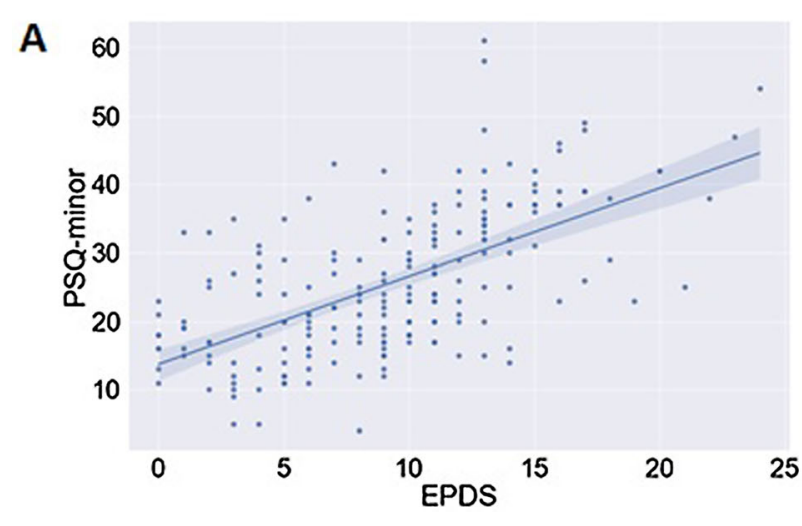

Fig. 3 Scatter plots showing the relationship between PSQ-C scores and EPDS scores. A Scatter plots showing the relationship between PSQ-C-minor score and EPDS

Average PSQ-C-minor Score

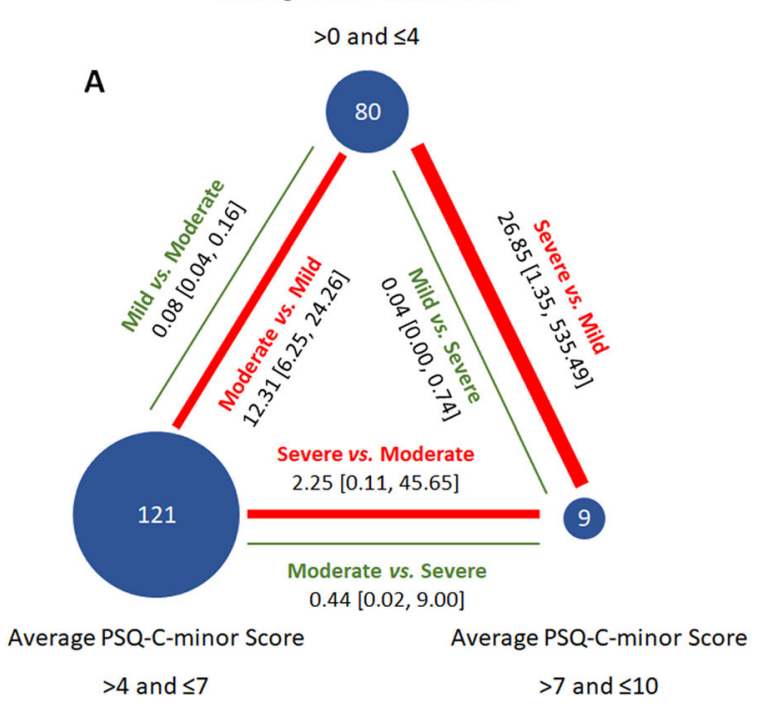

Fig. 4 Network geometry. Network of stratification of average PSQ-C-minor and PSQ-C-total scores; node size is proportional to the sample size in each stratified cluster and thickness of the lines (edges) in relation to the number of direct comparisons. Green lines indicate overall low risk

down the total effect of factors on PPD development into direct and indirect effects. The result indicated that postpartum pain sensitivity not only directly contributed to mothers having EPDS scores $\geq 10$ points, consistent with a previous study [41], but also indirectly influenced the incidence by decreasing breastfeeding rates. This relationship might arise

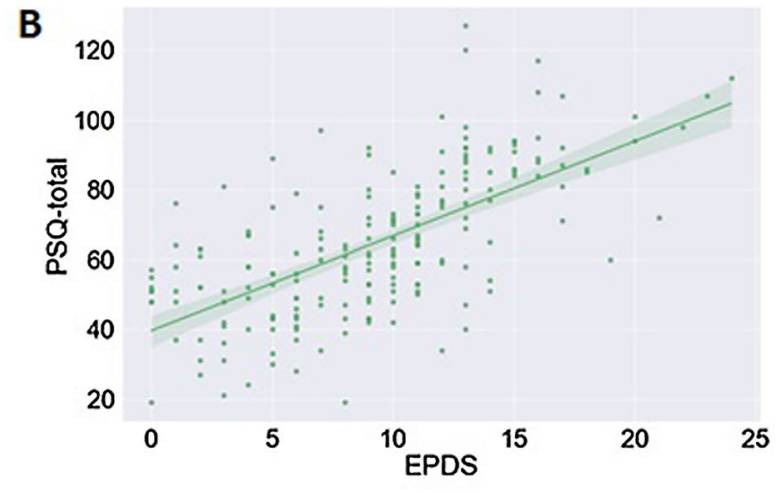

score; B scatter plots showing the relationship between PSQ-C-total score and EPDS score

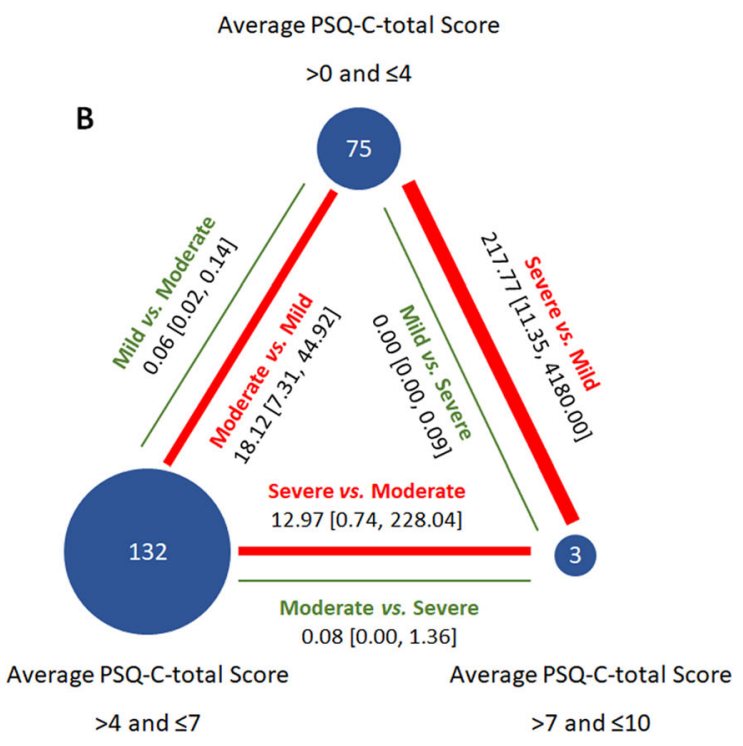

in the comparison, while red lines indicate high risk. A Network of stratification of average PSQ-C-minor scores; B network of stratification of average PSQ-C-total scores

because mothers experiencing increased postpartum pain sensitivity were more likely to refuse to breastfeed because of pain perception when their babies sucked their breast. Therefore, we highlight that postpartum pain management should be considered an important part of postnatal care service. 
Table 2 Summary of logistic regression analysis on positive PPD screen

\begin{tabular}{|c|c|c|}
\hline Variables & Crude OR $(95 \% \mathrm{CI})$ & $P$ value \\
\hline BMI & $1.008(0.929-1.095)$ & 0.845 \\
\hline Primipara & $0.740(0.426-1.284)$ & 0.284 \\
\hline \multicolumn{3}{|l|}{ Maternal disease } \\
\hline Hypertension & $1.429(0.281-7.251)$ & 0.667 \\
\hline Gestational diabetes & $2.364(1.097-5.094)$ & 0.028 \\
\hline Anemia & $1.115(0.552-2.251)$ & 0.761 \\
\hline \multicolumn{3}{|l|}{ Mode of delivery } \\
\hline Spontaneous & $0.630(0.362-1.097)$ & 0.102 \\
\hline Assisted vaginal & $2.081(0.800-5.400)$ & 0.133 \\
\hline Cesarean & $1.171(0.671-2.043)$ & 0.578 \\
\hline \multicolumn{3}{|l|}{ Stage of labor } \\
\hline First stage of labor & $1.001(0.999-1.002)$ & 0.338 \\
\hline Second stage of labor & $1.014(1.002-1.026)$ & 0.026 \\
\hline Total stage of labor & $1.001(1.000-1.002)$ & 0.174 \\
\hline Labor analgesia & $1.149(0.644-2.049)$ & 0.639 \\
\hline \multicolumn{3}{|l|}{ Perineal injury } \\
\hline I degree & $0.623(0.341-1.137)$ & 0.123 \\
\hline II degree & $0.621(0.208-1.856)$ & 0.393 \\
\hline III and IV degree & $1.984(0.976-4.033)$ & 0.058 \\
\hline Birth weight & $0.489(0.226-1.056)$ & 0.068 \\
\hline Exclusive breastfeeding & $0.339(0.148-0.777)$ & 0.011 \\
\hline PSQ-C-total score & $1.111(1.081-1.142)$ & $<0.001$ \\
\hline PSQ-C-minor score & $1.191(1.136-1.249)$ & $<0.001$ \\
\hline
\end{tabular}

$B M I$ body mass index, $O R$ odds ratio, $P P D$ postpartum depression, $P S Q-C$ Mandarin Chinese version of pain sensitivity questionnaire

Our study also showed that gestational diabetes could directly increase the incidence of mothers with EPDS scores $\geq 10$ points, which also corroborates previous findings [25]. Moreover, gestational diabetes also had an indirect effect on EPDS scores $\geq 10$ points through the mode of feeding. Although breastfeeding only

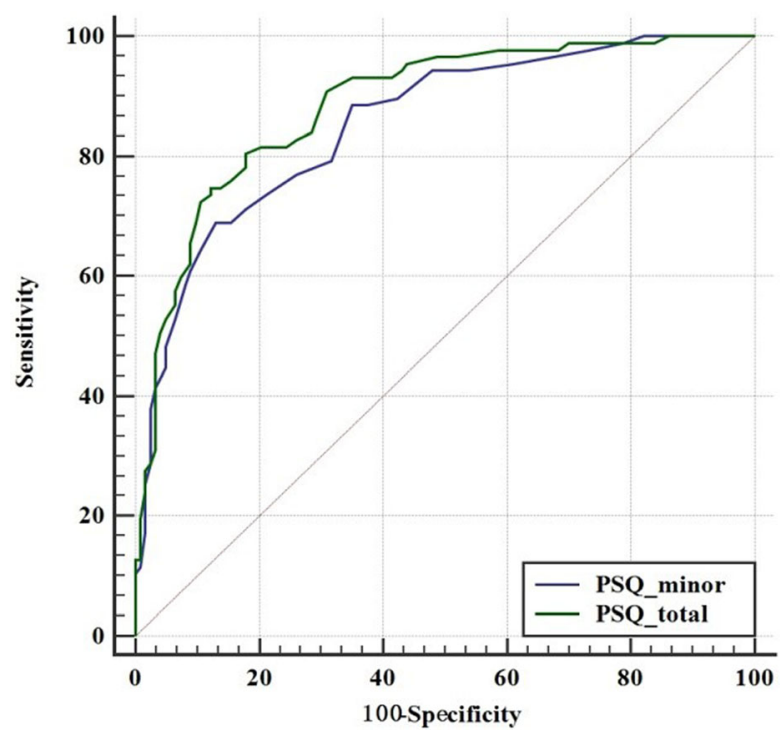

Fig. 5 Receiver-operating characteristic curves showing the ability of PSQ-C-total and PSQ-C-minor to predict PPD

had a direct effect on PPD, it played a critical and mediating role in other variables and PPD $[26,42]$. New emerging evidence showed an inverse relationship between breastfeeding and PPD [43], indicating that mothers with PPD generally had a negative experience with breastfeeding. However, breastfeeding has been confirmed to decrease PPD since it alleviates stress responses, decreases the serum cortisol level, and attenuates the psychological burden [44]. Besides, our study also revealed another factor, birth weight, had a direct effect on EPDS scores $\geq 10$ points, indicating that lower birth weight tended to cause mothers to develop PPD compared with normal birth weight $[45,46]$. The potential reasons birth weight has a direct effect on mothers' development of PPD can be concluded as follows: (1) poor growth of low birth weight babies concerned mothers; (2) low birth weight generally indicated low fetal age, and anticipated premature delivery pushed parturients into the mother role, leading to adaption insufficiency; (3) neonatal intensive care was necessary for low birth weight neonates, and maternal separation increased mothers' psychological burden.

Our study had several limitations. First, we only evaluated the influence of postpartum 
Table 3 Summary of multivariate logistic regression analysis on positive PPD screen

\begin{tabular}{lllc}
\hline Variables & Crude OR $(\mathbf{9 5 \%}$ CI) & Adjusted OR $(95 \%$ CI $)$ & $P$ value \\
\hline PSQ-C-total & $1.111(1.081-1.142)$ & $1.116(1.081-1.151)$ & $<0.0001$ \\
Gestational diabetes & $2.364(1.097-5.094)$ & $2.683(0.964-7.466)$ & 0.045 \\
Exclusive breastfeeding & $0.339(0.148-0.777)$ & $0.265(0.073-0.964)$ & 0.044 \\
Birth weight & $0.357(0.153-0.836)$ & $0.171(0.047-0.613)$ & 0.007 \\
\hline
\end{tabular}

$O R$ odds ratio, $P P D$ postpartum depression, $P S Q-C$ Mandarin Chinese version of pain sensitivity questionnaire

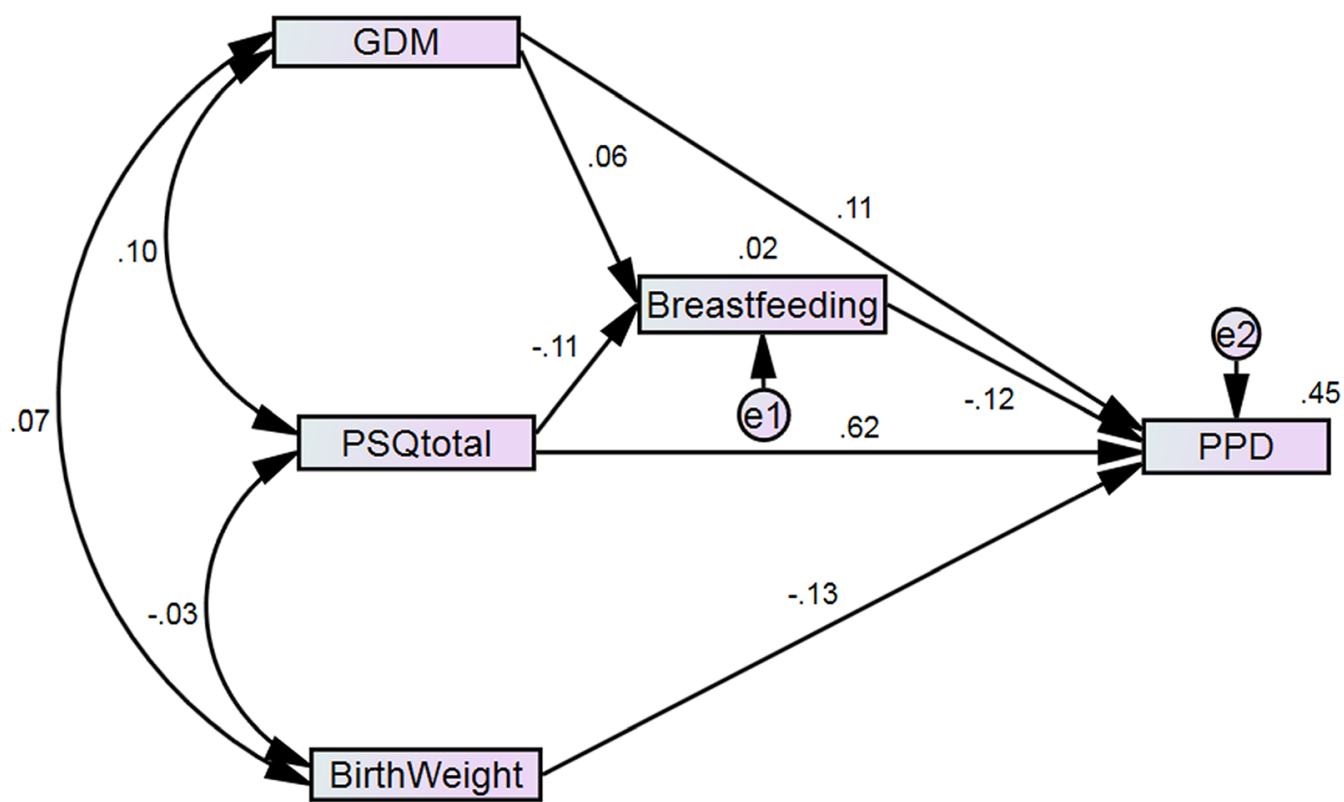

Fig. 6 Path analysis model showing the interconnections between risk factors and PPD

Table 4 Summary of the direct, indirect, and total effects on PPD by a path analysis model

\begin{tabular}{lrrr}
\hline Variables & \multicolumn{3}{l}{ Effects } \\
\cline { 2 - 4 } & \multicolumn{1}{l}{ Direct } & Indirect & \multicolumn{1}{c}{ Total } \\
\hline PSQ-C-total & 0.617 & 0.014 & 0.631 \\
Gestational diabetes & 0.107 & -0.007 & 0.100 \\
Exclusive breastfeeding & -0.120 & 0.000 & -0.120 \\
Birth weight & -0.125 & 0.000 & -0.125 \\
\hline
\end{tabular}

$P P D$ postpartum depression, PSQ-C Mandarin Chinese version of pain sensitivity questionnaire pain sensitivity and perinatal factors on EPDS scores $\geq 10$ points, and other potential confounding factors, such as socioeconomic status, emotional support from husbands and families, life stressors, and other negative stressful events, were not included. Second, the time point of our primary outcome was 6 weeks postpartum, and long-term follow-up for PPD was necessary. Third, the primary outcome focused on the predictive value of early increased postpartum pain sensitivity (1-week postpartum) on subsequent PPD (6-week postpartum), since this is the time of peak prevalence of PPD $[47,48]$ and because pain at this time after surgery correlates with chronic pain [49]. However, the longer term of postpartum hyperalgesia or chronic 
post-surgical pain (6 months postpartum) is also of great clinical importance and contributes to PPD. Therefore, the feature of postpartum pain, especially the later neuropathic component of the pain trajectory after delivery, needs to be clarified in the future. Fourth, although we conducted the sample size calculation prospectively based on subject-to-item and ratio of the questionnaire according to previous publications, however, a large sample size is still needed to confirm the results of mutifactorial regression analysis. Last, PPD was only screened by the EPDS scale ( $\geq 10$ points), and no objective tests of pain sensitivity were conducted in our study considering maternal comfort.

\section{CONCLUSIONS}

Our findings suggested that postpartum pain sensitivity was significantly associated with PPD screened by the EPDS scale ( $\geq 10$ points), and PSQ-C scores including PSQ-C-total and PSQ-Cminor scores would be a reliable predictor of PPD. We also identified several potential risk factors related to PPD including gestational diabetes, breastfeeding, and low birth weight. Moreover, we constructed a path analysis model to explore the potential interrelations between these factors and PPD. According to our findings, we highlighted that postpartum pain management should be considered in mothers during the peripartum period.

\section{ACKNOWLEDGEMENTS}

The authors thank all parturients who participated in the study and appreciate the efforts of the staff of the Department of Anesthesiology, Jinshan Branch of Fujian Provincial Hospital and Fujian Provincial Hospital in the project implementation and data collection. The authors also thank the master degree candidates from the Department of Anesthesiology, Jinshan Branch of Fujian Provincial Hospital, for their great contributions to data collection and data entry. Besides, the authors thank Haiping Zheng in the Core Facility of Biomedical,
Xiamen University, for her help with data review and analysis.

Funding. This work was partly supported by the National Natural Science Foundation of China (81701091, 81870828, 81801101), Joint Fund for Research and Development of Highlevel Hospital (2017LHJJ10), and Startup Fund for Scientific Research, Fujian Medical University (2020QH1155). The funders had no role in the study design, data collection and analysis, decision to publish, or preparation of the manuscript. The journal's Rapid Service Fee was funded by the authors.

Authorship. All named authors meet the International Committee of Medical Journal Editors (ICMJE) criteria for authorship for this article, take responsibility for the integrity of the work as a whole, and have given their approval for this version to be published.

Authors' Contributions. We fully acknowledge the participating investigators and active members in the project. Yu Chen and Huanghui $\mathrm{Wu}$ contributed to the concept and design of the research, collected data, and contributed to discussions and drafting of the manuscript. Xiaodan $\mathrm{Wu}$ contributed to the statistics and discussions and reviewed the results. All study participants' data were clinically assessed by $\mathrm{Yu}$ Chen and Xin Ye. Huanghui Wu and Xiaodan $\mathrm{Wu}$ are the guarantors of this work and, as such, had full access to all data in the study and take responsibility for the integrity of the data and the accuracy of the data analysis. Han $\mathrm{Wu}$, Xinlei Huang, Congrong Ke, and Yanqing Chen are investigators.

Disclosures. All authors (Yu Chen, Xin Ye, $\mathrm{Han} \mathrm{Wu}$, Xinlei Huang, Congrong Ke, Yanqing Chen, Huanghui $\mathrm{Wu}$, and Xiaodan $\mathrm{Wu}$ ) confirm that they have no conflicts of interest to disclose.

Compliance with Ethics Guidelines. All the procedures performed in the study involving human participants were in accordance with the ethical standards of the institutional and/or national research committee and with the 1964 
Helsinki Declaration and its later amendments or comparable ethical standards. The study protocol was approved by the Institutional Ethics Committee (IEC) of Fujian Provincial Hospital (no. K2020-04-047) and registered on ChiCTR.org.cn (ChiCTR-2000033091). All parturients received written and verbal information about the study from researchers or midwives, and they signed written consent forms before participation.

Data Availability. The authors declare that all data supporting the findings of this study are available within the article and from the corresponding author upon reasonable request.

Open Access. This article is licensed under a Creative Commons Attribution-NonCommercial 4.0 International License, which permits any non-commercial use, sharing, adaptation, distribution and reproduction in any medium or format, as long as you give appropriate credit to the original author(s) and the source, provide a link to the Creative Commons licence, and indicate if changes were made. The images or other third party material in this article are included in the article's Creative Commons licence, unless indicated otherwise in a credit line to the material. If material is not included in the article's Creative Commons licence and your intended use is not permitted by statutory regulation or exceeds the permitted use, you will need to obtain permission directly from the copyright holder. To view a copy of this licence, visit http://creativecommons.org/licenses/by$\mathrm{nc} / 4.0 /$.

\section{REFERENCES}

1. Shi P, Ren H, Li H, Dai Q. Maternal depression and suicide at immediate prenatal and early postpartum periods and psychosocial risk factors. Psychiatry Res. 2018;261:298-306.

2. Miksic S, Miskulin M, Juranic B, Rakosec Z, Vcev A, Degmecic D. Depression and suicidality during pregnancy. Psychiatr Danub. 2018;30:85-90.
3. Payne JL, Maguire J. Pathophysiological mechanisms implicated in postpartum depression. Front Neuroendocrinol. 2019;52:165-80.

4. Lindahl V, Pearson JL, Colpe L. Prevalence of suicidality during pregnancy and the postpartum. Arch Womens Ment Health. 2005;8:77-87.

5. Liang P, Wang Y, Shi S, Liu Y, Xiong R. Prevalence and factors associated with postpartum depression during the COVID-19 pandemic among women in Guangzhou, China: a cross-sectional study. BMC Psychiatry. 2020;20:557.

6. Gao M, Hu J, Yang L, Ding N, Wei X, Li L, et al. Association of sleep quality during pregnancy with stress and depression: a prospective birth cohort study in China. BMC Pregnancy Childbirth. 2019;19:444.

7. Liu Y, Guo N, Li T, Zhuang W, Jiang H. Prevalence and associated factors of postpartum anxiety and depression symptoms among women in Shanghai. China J Affect Disord. 2020;274:848-56.

8. Stewart DE, Vigod SN. Postpartum depression: pathophysiology, treatment, and emerging therapeutics. Annu Rev Med. 2019;70:183-96.

9. Liu TT, Raju A, Boesel T, Cyna AM, Tan SG. Chronic pain after caesarean delivery: an Australian cohort. Anaesth Intensive Care. 2013;41:496-500.

10. Wang LZ, Wei CN, Xiao F, Chang XY, Zhang YF. Incidence and risk factors for chronic pain after elective caesarean delivery under spinal anaesthesia in a Chinese cohort: a prospective study. Int J Obstet Anesth. 2018;34:21-7.

11. Jin J, Peng L, Chen Q, Zhang D, Ren L, Qin P, et al. Prevalence and risk factors for chronic pain following cesarean section: a prospective study. BMC Anesthesiol. 2016;16:99.

12. Eisenach JC, Pan PH, Smiley R, Lavand'homme P, Landau R, Houle TT. Severity of acute pain after childbirth, but not type of delivery, predicts persistent pain and postpartum depression. Pain. 2008;140:87-94.

13. Maldonado RJ, De Jesus O. Hyperesthesia. StatPearls. Treasure Island (FL) 2021.

14. Quesada C, Kostenko A, Ho I, Leone C, Nochi Z, Stouffs A, et al. Human surrogate models of central sensitization: a critical review and practical guide. Eur J Pain. 2021;25:1389-428.

15. Glare P, Aubrey KR, Myles PS. Transition from acute to chronic pain after surgery. Lancet (London, England). 2019;393:1537-46. 
16. Althaus A, Arránz Becker O, Neugebauer E. Distinguishing between pain intensity and pain resolution: using acute post-surgical pain trajectories to predict chronic post-surgical pain. Eur J Pain (London, England). 2014;18:513-21.

17. Bair MJ, Robinson RL, Katon W, Kroenke K. Depression and pain comorbidity: a literature review. Arch Intern Med. 2003;163:2433-45.

18. Conejero I, Olié E, Calati R, Ducasse D, Courtet P. Psychological pain, depression, and suicide: recent evidences and future directions. Curr Psychiatry Rep. 2018;20:33.

19. Ruscheweyh R, Verneuer B, Dany K, Marziniak M, Wolowski A, Colak-Ekici R, et al. Validation of the pain sensitivity questionnaire in chronic pain patients. Pain. 2012;153:1210-8.

20. Tuna T, Van Obbergh L, Van Cutsem N, Engelman E. Usefulness of the pain sensitivity questionnaire to discriminate the pain behaviour of chronic pain patients. Br J Anaesth. 2018;121:616-22.

21. Grundström H, Larsson B, Arendt-Nielsen L, Gerdle B, Kjølhede P. Associations between pain thresholds for heat, cold and pressure, and Pain Sensitivity Questionnaire scores in healthy women and in women with persistent pelvic pain. Eur J Pain (London, England). 2019;23:1631-9.

22. Quan X, Fong DYT, Leung AYM, Liao Q, Ruscheweyh R, Chau PH. Validation of the mandarin chinese version of the pain sensitivity questionnaire. Pain Pract. 2018;18:180-93.

23. Ruscheweyh R, Marziniak M, Stumpenhorst F, Reinholz J, Knecht S. Pain sensitivity can be assessed by self-rating: development and validation of the Pain Sensitivity Questionnaire. Pain. 2009;146: 65-74.

24. Cox JL, Holden JM, Sagovsky R. Detection of postnatal depression. Development of the 10-item Edinburgh postnatal depression scale. Br J Psychiatry. 1987;150:782-6.

25. Hinkle SN, Buck Louis GM, Rawal S, Zhu Y, Albert PS, Zhang C. A longitudinal study of depression and gestational diabetes in pregnancy and the postpartum period. Diabetologia. 2016;59:2594-602.

26. Liu S, Yan Y, Gao X, Xiang S, Sha T, Zeng G, et al. Risk factors for postpartum depression among Chinese women: path model analysis. BMC Pregnancy Childbirth. 2017;17:133.

27. Lim G, Farrell LM, Facco FL, Gold MS, Wasan AD. Labor analgesia as a predictor for reduced postpartum depression scores: a retrospective observational study. Anesth Analg. 2018;126:1598-605.
28. DeLong ER, DeLong DM, Clarke-Pearson DL. Comparing the areas under two or more correlated receiver operating characteristic curves: a nonparametric approach. Biometrics. 1988;44:837-45.

29. McDonald RP, Ho MH. Principles and practice in reporting structural equation analyses. Psychol Methods. 2002; 7:64-82.

30. Breivik H, Borchgrevink PC, Allen SM, Rosseland LA, Romundstad L, Hals EK, et al. Assessment of pain. Br J Anaesth. 2008;101:17-24.

31. Street LM, Harris L, Curry RS, Eisenach JC. Capsaicin-induced pain and sensitisation in the postpartum period. Br J Anaesth. 2019;122:103-10.

32. Niklasson B, Georgsson Ohman S, Segerdahl M, Blanck A. Risk factors for persistent pain and its influence on maternal wellbeing after cesarean section. Acta Obstet Gynecol Scand. 2015;94:622-8.

33. Kainu JP, Halmesmaki E, Korttila KT, Sarvela PJ. Persistent pain after cesarean delivery and vaginal delivery: a prospective cohort study. Anesth Analg. 2016;123:1535-45.

34. Kainu JP, Sarvela J, Tiippana E, Halmesmaki E, Korttila KT. Persistent pain after caesarean section and vaginal birth: a cohort study. Int J Obstet Anesth. 2010;19:4-9.

35. Karlstrom A, Engstrom-Olofsson R, Norbergh KG, Sjoling M, Hildingsson I. Postoperative pain after cesarean birth affects breastfeeding and infant care. J Obstet Gynecol Neonatal Nurs. 2007;36:430-40.

36. Swenson CW, DePorre JA, Haefner JK, Berger MB, Fenner DE. Postpartum depression screening and pelvic floor symptoms among women referred to a specialty postpartum perineal clinic. Am J Obstet Gynecol. 2018;218:335.

37. Soet JE, Brack GA, DiIorio C. Prevalence and predictors of women's experience of psychological trauma during childbirth. Birth (Berkeley, Calif). 2003;30:36-46.

38. Kim HJ, Lee JI, Kang KT, Chang BS, Lee CK, Ruscheweyh R, et al. Influence of pain sensitivity on surgical outcomes after lumbar spine surgery in patients with lumbar spinal stenosis. Spine. 2015;40:193-200.

39. Azimi P, Benzel EC. Cut-off value for pain sensitivity questionnaire in predicting surgical success in patients with lumbar disc herniation. PLoS ONE. 2016;11:e0160541.

40. Kil HK, Kim WO, Chung WY, Kim GH, Seo H, Hong JY. Preoperative anxiety and pain sensitivity are independent predictors of propofol and sevoflurane 
requirements in general anaesthesia. Br J Anaesth. 2012;108:119-25.

41. Babazade R, Vadhera RB, Krishnamurthy P, Varma A, Doulatram G, Saade GR, et al. Acute postcesarean pain is associated with in-hospital exclusive breastfeeding, length of stay and post-partum depression. J Clin Anesth. 2020;62:109697.

42. Watkins S, Meltzer-Brody S, Zolnoun D, Stuebe A. Early breastfeeding experiences and postpartum depression. Obstet Gynecol. 2011;118:214-21.

43. Webber E, Benedict J. Postpartum depression: a multi-disciplinary approach to screening, management and breastfeeding support. Arch Psychiatr Nurs. 2019;33:284-9.

44. Figueiredo B, Dias CC, Brandão S, Canário C, Nunes-Costa R. Breastfeeding and postpartum depression: state of the art review. Jornal de Pediatria. 2013;89:332-8.

45. Helle N, Barkmann C, Bartz-Seel J, Diehl T, Ehrhardt $S$, Hendel A, et al. Very low birth-weight as a risk factor for postpartum depression four to six weeks postbirth in mothers and fathers: cross-sectional results from a controlled multicentre cohort study. J Affect Disord. 2015;180:154-61.

46. Vigod SN, Villegas L, Dennis CL, Ross LE. Prevalence and risk factors for postpartum depression among women with preterm and low-birth-weight infants: a systematic review. BJOG. 2010;117: 540-50.

47. Patel RR, Murphy DJ, Peters TJ. Operative delivery and postnatal depression: a cohort study. BMJ. 2005;330:879.

48. Wisner KL, Parry BL, Piontek CM. Clinical practice. Postpartum depression. N Engl J Med. 2002;347: 194-9.

49. Kehlet H, Jensen TS, Woolf CJ. Persistent postsurgical pain: risk factors and prevention. Lancet. 2006;367:1618-25. 